\title{
Genetic testing for inherited heart diseases: longitudinal impact on health-related quality of life
}

\author{
Jodie Ingles, GradDipGenCouns, PhD 1,2, Laura Yeates, BSc, GradDipGenCouns', \\ Lisa O'Brien, BSc, MSc (GenCouns) ${ }^{3}$ Julie McGaughran, MD ${ }^{3,4}$, Paul A. Scuffham, BA, PhD5, \\ John Atherton, MBBS, PhD ${ }^{4,6}$ and Christopher Semsarian, MBBS, PhD ${ }^{1,2,7}$
}

\begin{abstract}
Purpose: A genetic diagnosis is an extremely useful tool in the management and care of families with inherited heart diseases, particularly in allowing clarification of risk status of asymptomatic family members. The psychosocial consequences of genetic testing in this group are poorly understood. This longitudinal pilot study sought to determine changes in health-related quality of life in patients and asymptomatic family members undergoing genetic testing for inherited heart diseases.
\end{abstract}

Methods: Individuals attending two specialized multidisciplinary cardiac genetic clinics in Australia were invited to participate. Patients undergoing proband or predictive genetic testing for an inherited cardiomyopathy or primary arrhythmogenic disorder were eligible. The Medical Outcomes Short Form-36 (version 2) was administered before the genetic result was given, and follow-up surveys were completed $1-3,6$, and 12 months after the result was given.

\section{INTRODUCTION}

There are now more than 40 cardiovascular disorders in which a genetic cause has been identified, including the inherited cardiomyopathies such as hypertrophic cardiomyopathy (HCM), familial dilated cardiomyopathy (FDC), and arrhythmogenic right ventricular cardiomyopathy, and primary arrhythmogenic disorders including long QT syndrome (LQTS). Even though these diseases are clinically and genetically distinct, they share many common genetic counseling issues..$^{1,2}$ These include autosomal dominant inheritance, marked variability in onset and severity of symptoms, lifetime periodic clinical surveillance of at-risk family members, and risk of sudden cardiac death..$^{3-6}$ Genetic testing is available for these diseases, and although a genetic diagnosis does offer clarification of risk status, limitations of testing include less-than-ideal mutation detection rates and limited access to testing due to costs.

Health-related quality of life (HR-QoL) is increasingly recognized as an important outcome measure in cardiac diseases, with many studies finding it to be predictive of mortality,
Results: A total of 54 individuals with hypertrophic cardiomyopathy, familial dilated cardiomyopathy, arrhythmogenic right ventricular cardiomyopathy, and long QT syndrome completed baseline and at least one follow-up survey, including 33 probands and 21 asymptomatic relatives. Physical and mental component scores analyzed at baseline and 1-3 months were found to be unchanged in all groups. Furthermore, no significant differences were observed up to 12 months after result.

Conclusion: In this longitudinal pilot study, no change in healthrelated quality of life was observed up to 12 months after the result was given in patients and their asymptomatic family members undergoing genetic testing for an inherited heart disease.

Genet Med 2012:14(8):749-752.

Key Words: genetic testing; health-related quality of life; hypertrophic cardiomyopathy; long QT syndrome

hospital readmission, and health-care costs. ${ }^{7}$ Predictive genetic testing of asymptomatic individuals at risk for disease can elicit a number of psychosocial responses, and understanding these has been an important research focus. ${ }^{8-10}$ Fear and concern that predictive genetic testing will have a negative impact on psychological state is largely unfounded based on studies carried out in cancer and neurologic genetic diseases..$^{8-10}$ In cardiac genetics, the data are limited with only a small number of studies investigating the impact of genetic testing. ${ }^{1,11-14}$ Understanding the psychosocial consequences of cardiac genetic testing can help to tailor pretest genetic counseling to best meet the needs of the patient.

This prospective, longitudinal pilot study sought to examine the HR-QoL of individuals undergoing genetic testing for inherited heart diseases. Specifically, the aim of this study was to identify whether there were changes in HR-QoL following genetic testing for an inherited heart disease in patients with clinical disease and their asymptomatic family members.

\footnotetext{
${ }^{1}$ Agnes Ginges Centre for Molecular Cardiology, Centenary Institute, Sydney, Australia; ${ }^{2}$ Sydney Medical School, University of Sydney, Sydney, Australia; ${ }^{3}$ Genetic Health Queensland, Royal Brisbane and Women's Hospital, Brisbane, Australia; ${ }^{4}$ School of Medicine, University of Queensland, Brisbane, Australia; ${ }^{5} \mathrm{Centre}$ for Applied Health Economics, School of Medicine, Griffith University, Brisbane, Australia; ${ }^{6}$ Department of Cardiology, Royal Brisbane and Women's Hospital, Brisbane, Australia; ${ }^{7}$ Department of Cardiology, Royal Prince Alfred Hospital, Sydney, Australia. Correspondence: Christopher Semsarian (c.semsarian@centenary.org.au)
} 


\section{MATERIALS AND METHODS}

\section{Patients}

Patients attending the Hypertrophic Cardiomyopathy and Genetic Heart Disease Clinics, Royal Prince Alfred Hospital in Sydney, Australia, and the Cardiac Genetics Clinic, Royal Brisbane and Women's Hospital in Brisbane, Australia, were invited to participate. These are specialized cardiac genetic clinics incorporating a multidisciplinary approach to care, and offer the services of a cardiologist, a clinical geneticist, and a genetic counselor. ${ }^{1,3,12}$

Inclusion criteria were as follows: clinically affected individuals and first-degree relatives at risk of a genetic heart disease aged more than 15 years. Diseases included the inherited cardiomyopathies such as HCM, FDC, arrhythmogenic right ventricular cardiomyopathy, and the primary arrhythmogenic disorder, LQTS. The respective institutional human research ethics committees approved all aspects of this study.

\section{Health status evaluation}

Health status was evaluated using the Medical Outcomes Survey Short Form-36, version 2. ${ }^{15,16}$ This is one of the most widely used measures of HR-QoL in the world and its validity has been shown in many populations, including Australia., ${ }^{17}$ The Short Form-36 measures eight dimensions of health and can be combined to produce two summary measures providing overall estimates for physical health (physical component score (PCS)) and mental health (mental component score (MCS)). Clinical information relating to disease status was collected from both the National Genetic Heart Disease Registry ${ }^{18}$ and available medical records.

The surveys were administered longitudinally, with a baseline survey being collected before the genetic result was given, and follow-up surveys being conducted at 1-3, 6 , and 12 months after the result was given. Participants who consented to the study and completed a baseline survey but failed to return subsequent surveys were deemed "nonresponders."

Table 1 Characteristics of the study cohort

\begin{tabular}{lcccc} 
& Group 1 & Group 2 & Group 3 & Group 4 \\
\hline Testing & Proband & Proband & Predictive & Predictive \\
Gene result & Positive & Indeterminate & Positive & Negative \\
N(\%) & $17(31)$ & $16(30)$ & $7(13)$ & $14(26)$ \\
$\begin{array}{l}\text { Age, years } \\
\text { (SD) }\end{array}$ & $46(13)$ & $52(19)$ & $40(10)$ & $48(13)$ \\
$\begin{array}{l}\text { Gender, } \\
\text { male }(\%)\end{array}$ & $8(47)$ & $10(63)$ & $2(29)$ & $5(36)$ \\
HCM & $11(65)$ & $10(63)$ & $6(86)$ & $8(57)$ \\
LQTS & $1(6)$ & $5(31)$ & - & $1(7)$ \\
ARVC & $4(24)$ & $1(6)$ & - & $1(7)$ \\
FDC & $1(6)$ & 0 & $1(14)$ & $4(29)$ \\
\hline
\end{tabular}

ARVC, arrhythmogenic right ventricular cardiomyopathy; FDC, familial dilated cardiomyopathy; HCM, hypertrophic cardiomyopathy; LQTS, long QT syndrome.

\section{Statistical analysis}

Data were analyzed using Prism (version 5.0; GraphPad, La Jolla, CA). The primary outcome measures were the PCS and MCS; these were converted to Australian weighted T-scores where scores range from 0 (worst possible health) to 100 (best possible health), with 50 as the mean score for the general Australian population. ${ }^{19}$ The primary analysis investigated a difference between baseline and time point 1 (1-3 months) scores, and for this paired $t$-tests were used. Further time points were analyzed using one-way analysis of variance to look for differences between the baseline and additional follow-up time points. A $P$ value $<0.05$ was considered statistically significant. Nonresponder baseline PCS and MCS were compared with clinically affected or asymptomatic baseline surveys using standard $t$ tests.

\section{RESULTS}

\section{Population characteristics}

A total of 54 individuals were recruited to this study, including 33 clinically affected probands and 21 asymptomatic family members (Table 1). In total, 21 probands had a diagnosis of HCM, 6 of LQTS, 5 of arrhythmogenic right ventricular cardiomyopathy, and 1 of FDC. The proband group included 17 individuals who received a genetic diagnosis following genetic testing (Group 1; mean age $48 \pm 13$ years, $47 \%$ males) and 16 who had no gene mutation identified after genetic testing (Group 2), also termed an indeterminate genetic test result (mean age $52 \pm 19$ years, 63\% males).

Of the asymptomatic relatives, seven were identified as gene carriers (Group 3; mean age $40 \pm 10$ years, 29\% males) and 14 were found not to carry the family gene mutation (Group 4; mean age $48 \pm 13$ years, $36 \%$ males). In this group, 14 were at risk of HCM, 5 of FDC, 1 of LQTS, and 1 of arrhythmogenic right ventricular cardiomyopathy.

\section{PCS and MCS in the proband group}

The PCS and MCS in the proband group are shown in Table 2. There was no significant difference between baseline and time point 1 (1-3 months) scores in the proband groups who received a genetic diagnosis (Group 1) or an indeterminate genetic result (Group 2). No significant changes in scores were observed up to 12 months after result.

\section{PCS and MCS in the asymptomatic individuals}

The PCS and MCS in the asymptomatic individuals are shown in Table 2. There was no significant difference between the baseline and time point 1 (1-3 months) scores in the asymptomatic groups who received a predictive positive (Group 3 ) and predictive negative (Group 4) genetic result. No significant changes in scores were observed up to 12 months after genetic testing.

\section{DISCUSSION}

This prospective, longitudinal pilot study evaluated the health status of clinically affected and asymptomatic individuals undergoing genetic testing for a variety of inherited heart diseases. 


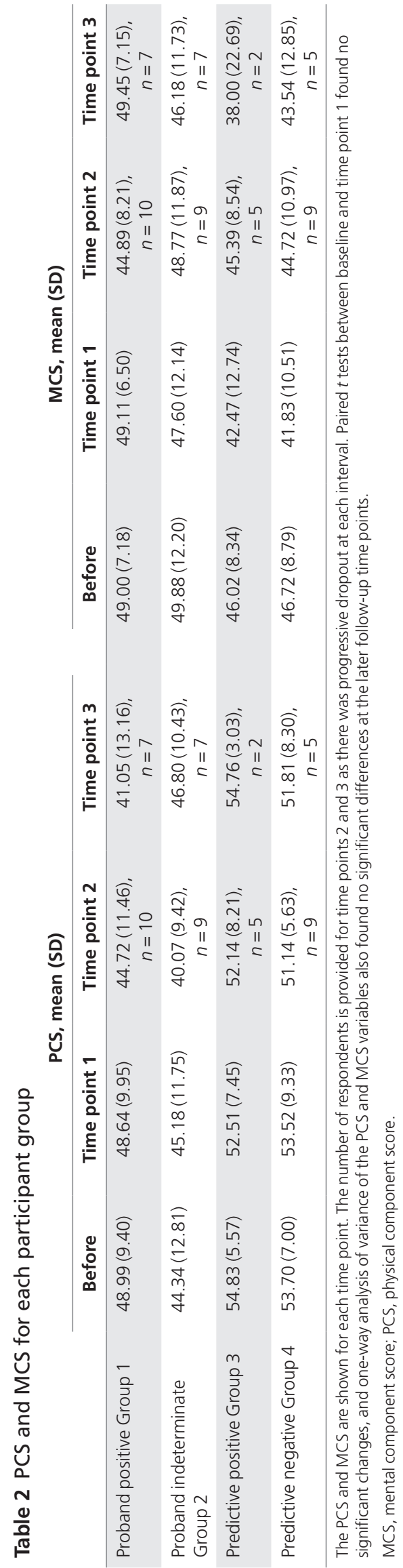

Although this was a pilot study, the preliminary results suggest that there is no change in HR-QoL following either proband or predictive genetic testing, and this outcome is maintained 12 months after result.

Given the clinical benefit of receiving a negative predictive gene result, it is not surprising that Group 4 showed no impaired HR-QoL. However, our results also suggest that the asymptomatic family members who receive a positive genetic result likewise show no change in HR-QoL. A positive predictive gene result implies a greater lifetime risk of developing disease, even though some level of uncertainty remains where clinically unaffected gene carriers are increasingly reported. ${ }^{20,21}$ Hence, this group is advised to undergo more frequent and rigorous clinical surveillance. The psychological impact on asymptomatic gene carriers has been extensively studied in familial cancer and neurological disease patients, and likewise no significant effect on emotional well-being or HR-QoL has been reported to date. ${ }^{8-10}$ Adaptation to the shift in health status, as well as the advantages of a gain in knowledge and more accurate risk perception have all been suggested to account for this lack of psychological harm following a positive predictive result, and this is well described in the literature. ${ }^{22,23}$ Of note, both proband groups (Groups 1 and 2) showed low HR-QoL scores, reflecting the fact they have clinical evidence of disease. ${ }^{1}$

Specifically in cardiology, limited data exist about the impact of genetic testing for inherited heart diseases. Cross-sectional studies investigating asymptomatic HCM mutation carriers suggest HR-QoL or psychological well-being is maintained. ${ }^{1,11}$ A longitudinal follow-up study of LQTS patients after predictive gene testing found elevated psychological distress initially, but this began to return to baseline in the long-term, unless the patient was also found to have an abnormal electrocardiogram, in which case the higher levels of distress persisted. ${ }^{14}$ Small studies have also been conducted in children undergoing genetic testing. One such study qualitatively described the outcomes of children who had undergone predictive genetic testing for LQTS, HCM, and familial hypercholesterolemia and found these children to have positive future health perceptions. ${ }^{24}$ Of note, a study assessing the impact of genetic testing in children in the context of the family found parents of LQTS children rated their child's psychological well-being as impaired, when in reality their scores were comparable to the general pediatric population. ${ }^{25}$ Our data, therefore, aligns to the limited literature to date suggesting that there is no change in psychosocial and health status outcome following genetic testing for inherited heart diseases.

The role of genetic counseling as an integral component of the pre- and posttest discussions should be acknowledged, specifically in cardiovascular diseases. ${ }^{2}$ In Australia, cardiac genetic testing is offered through clinical genetics departments or specialized cardiac genetic clinics, which adopt a multidisciplinary approach including genetic counseling. ${ }^{3,12}$ Of note, genetic counseling has been shown in a number of studies to promote psychological well-being through educational and emotional support, including improved psychosocial responses 
of patients who regularly attend this type of multidisciplinary clinic. ${ }^{12,26}$ Participants in this study were recruited directly from two specialized clinics incorporating a multidisciplinary approach to care and this may represent a better adjusted and more informed cohort.

Nonresponders made up only a small percentage of participants $(n=8)$, however baseline comparison of HR-QoL scores was performed to assess whether this group who failed to return any subsequent follow-up surveys represented different health status outcomes. Although the nonresponders were a small group, no significant difference was observed between asymptomatic and clinically affected nonresponders.

In conclusion, this prospective, longitudinal pilot study suggests that both probands and asymptomatic family members had no change in HR-QoL up to 12 months after result. Future studies based on larger patient cohorts undergoing genetic testing must be carried out using additional measures of psychosocial status. A larger patient population will allow more powerful repeated-measures analyses to be applied, meaning necessary subgroup analyses can be performed, including assessment of the psychosocial impact of multiple mutation genotypes, variants of uncertain significance, and predictive genetic testing in children. Results from further studies have the potential to inform clinical management strategies in patients' receiving genetic testing results, including improved education, increased awareness, and access to genetic counseling services.

\section{ACKNOWLEDGMENTS}

J.I. is the recipient of a University of Sydney Postgraduate Award, and C.S. is the recipient of a National Health and Medical Research Council (NHMRC) Practitioner Fellowship. This study was also supported, in part, by an NHMRC project grant.

\section{DISCLOSURE}

The authors declare no conflict of interest.

\section{REFERENCES}

1. Ingles J, Yeates L, Hunt L, et al. Health status of cardiac genetic disease patients and their at-risk relatives. Int J Cardio/ 2011; e-pub ahead of print 17 September 2011.

2. Ingles J, Yeates L, Semsarian C. The emerging role of the cardiac genetic counselor. Heart Rhythm 2011;8:1958-1962.

3. Ingles J, Semsarian C. Sudden cardiac death in the young: a clinical genetic approach. Intern Med J 2007;37:32-37.

4. Christiaans I, van Langen IM, Birnie E, Bonsel GJ, Wilde AA, Smets EM Genetic counseling and cardiac care in predictively tested hypertrophic cardiomyopathy mutation carriers: the patients' perspective. Am J Med Genet A 2009;149A:1444-1451.

5. Hershberger RE, Cowan J, Morales A, Siegfried JD. Progress with genetic cardiomyopathies: screening, counseling, and testing in dilated, hypertrophic, and arrhythmogenic right ventricular dysplasia/ cardiomyopathy. Circ Heart Fail 2009;2:253-261.

6. Ingles J, Doolan A, Chiu C, Seidman J, Seidman C, Semsarian C. Compound and double mutations in patients with hypertrophic cardiomyopathy: implications for genetic testing and counselling. J Med Genet 2005;42:e59.

7. Konstam V, Salem D, Pouleur H, et al. Baseline quality of life as a predictor of mortality and hospitalization in 5,025 patients with congestive heart failure. SOLVD Investigations. Studies of Left Ventricular Dysfunction Investigators. Am J Cardiol 1996;78:890-895.

8. Broadstock M, Michie S, Marteau T. Psychological consequences of predictive genetic testing: a systematic review. Eur J Hum Genet 2000;8:731-738.

9. Prévost $C$, Veillette $S$, Perron $M$, et al. Psychosocial impact of predictive testing for myotonic dystrophy type 1. Am J Med Genet A 2004;126A:68-77.

10. Tibben A. Predictive testing for Huntington's disease. Brain Res Bull 2007;72:165-171.

11. Christiaans I, van Langen IM, Birnie E, Bonsel GJ, Wilde AA, Smets EM. Quality of life and psychological distress in hypertrophic cardiomyopathy mutation carriers: a cross-sectional cohort study. Am J Med Genet A 2009;149A:602-612.

12. Ingles J, Lind JM, Phongsavan P, Semsarian C. Psychosocial impact of specialized cardiac genetic clinics for hypertrophic cardiomyopathy. Genet Med 2008;10:117-120.

13. van Maarle MC, Stouthard ME, Bonsel GJ. Quality of life in a family based genetic cascade screening programme for familial hypercholesterolaemia: a longitudinal study among participants. J Med Genet 2003;40:e3.

14. Hendriks KS, Hendriks MM, Birnie E, et al. Familial disease with a risk of sudden death: a longitudinal study of the psychological consequences of predictive testing for long QT syndrome. Heart Rhythm 2008;5:719-724.

15. Ware JE, Kosinski M. Interpreting SF-36 summary health measures: a response. Qual Life Res 2001;10:405-13; discussion 415.

16. Ware JE Jr, Sherbourne CD. The MOS 36-item short-form health survey (SF-36). I. Conceptual framework and item selection. Med Care 1992;30:473-483.

17. McCallum J. The SF-36 in an Australian sample: validating a new, generic health status measure. Aust J Public Health 1995;19:160-166.

18. Ingles J, McGaughran J, Vohra J, et al. Establishment of an Australian National Genetic Heart Disease Registry. Heart Lung Circ 2008;17:463467.

19. Hawthorne G, Osborne RH, Taylor A, Sansoni J. The SF36 Version 2: critical analyses of population weights, scoring algorithms and population norms. Qual Life Res 2007;16:661-673.

20. Maron BJ, Yeates L, Semsarian C. Clinical challenges of genotype positive (+)-phenotype negative (-) family members in hypertrophic cardiomyopathy. Am J Cardio/ 2011;107:604-608.

21. Gray B, Ingles J, Semsarian C. Natural history of genotype positivephenotype negative patients with hypertrophic cardiomyopathy. Int J Cardio/ 2011;152:258-259.

22. Stiggelbout AM, de Vogel-Voogt E. Health state utilities: a framework for studying the gap between the imagined and the real. Value Health 2008;11:76-87.

23. Schwartz CE, Bode R, Repucci N, Becker J, Sprangers MA, Fayers PM. The clinical significance of adaptation to changing health: a meta-analysis of response shift. Qual Life Res 2006;15:1533-1550.

24. Meulenkamp TM, Tibben A, Mollema ED, et al. Predictive genetic testing for cardiovascular diseases: impact on carrier children. Am J Med Genet $A$ 2008;146A:3136-3146.

25. Smets EM, Stam MM, Meulenkamp TM, et al. Health-related quality of life of children with a positive carrier status for inherited cardiovascular diseases. Am J Med Genet A 2008;146A:700-707.

26. Biesecker BB. Goals of genetic counseling. Clin Genet 2001;60:323-330. 